\title{
versants
}

\section{La règle du jeu}

Michèle Crogiez Labarthe

Université de Berne

\begin{abstract}
: À l'époque classique du moins, en vers comme en prose, les textes obéissent à toutes sortes de contraintes : celles qu'imposent le thème mais également le genre littéraire, les conventions sociales, les attentes du public plus ou moins large auquel l'auteur s'adresse, voire les lois en vigueur. Mais ces contraintes dans toutes leurs combinatoires peuvent produire bien des effets de sens d'une grande variété. Plus les genres sont codés, plus le jeu littéraire consiste à innover, autrement dit à jouer avec les règles, que ce soit sereinement, avec ironie, voire sur le mode de la dénonciation, pour contourner toutes les formes de censure.
\end{abstract}

Keywords: Littérature, liberté, censure, sous-entendu, genre littéraire.

"Qui ne voit que ceci est jeu, et par conséquent ne peut porter coup?»

La Fontaine, Contes, Préface, I665.

Qui pense littérature "classique » comme on dit aujourd'hui, pense souvent à une littérature normée, codifiée, ployant sous les exigences du canon rhétorique ou stylistique que les auteurs de cette époque auraient acceptées avec un respect soigneux. Peut-être est-ce d'ailleurs là l'hommage que certains d'entre eux attendaient de la postérité en reconnaissance de leurs efforts. Mais il est permis d'en douter, au moins pour les plus alertes, si on observe toutes les ruses par lesquelles les auteurs tournent, à l'occasion, les règles contre elles-mêmes. Si pesante qu'elle puisse avoir été dans le contexte monarchique de la France des $\mathrm{XVII}^{\mathrm{e}}$ et XVIII ${ }^{\mathrm{e}}$ siècles, la rigueur avec laquelle codes et conventions littéraires sont respectés est la forêt où se cachent quelques essences inattendues. Il ne s'agit nullement pour les collaborateurs de ce numéro d'ignorer ou de dédaigner la force des codes de toute nature qui régissaient les genres littéraires et la bienséance puisque c'est justement leur rigueur qui a suscité, paradoxalement, l'envie de les contourner ou de les subvertir. Il ne s'agit ici ni plus ni moins que d'observer l'espace de liberté que les auteurs savent aménager en vers et en prose, au cœur même du système littéraire, et même en dehors des institutions littéraires telles que les académies, pour donner à entendre autre chose que l'explicite de leur discours. La littérature se révèle bien être, si l'occasion le permet et surtout quand elle paraît ne pas devoir l'autoriser, un discours oblique : capable de répondre à titre principal à un thème, à une ambition, voire à un cérémonial 
donné mais, subrepticement, capable aussi d'exprimer un message plus caché et plus profond que l'auteur offre à qui saura l'entendre.

Il ne s'agit certes pas, pour l'auteur, de se rendre incompréhensible ! Le message ambigu, caché, dissimulé, doit pouvoir être mis au jour, fût-ce par peu de lecteurs. Or le temps passant, certaines conditions de compréhension s'émoussent, disparaissent, deviennent presque inenvisageables. L'aventure survenue au De incertitudine et vanitate omnium scientiarum de Cornelius Agrippa (1527) en donne un bon exemple : après des querelles de spécialistes supputant pourquoi le grand savant humaniste dénonçait la vanité des sciences, en contradiction avec tout le reste de son œuvre et par une incohérence impossible à justifier aux yeux des modernes, Barbara Bowen a montré que la méconnaissance contemporaine du fonctionnement de l'éloge paradoxal en portait toute la responsabilité. Un humaniste assuré de la valeur du savoir n'a en effet aucune peine à se donner l'exercice rafraîchissant de développer un plaidoyer charpenté en faveur de l'ignorance sans qu'on doive y entendre plus qu'un jeu récréatif, qui n'exprime strictement aucune mise en cause existentielle du dévouement inébranlable qu'il porte à la science et à la connaissance. Pour que certains messages reprennent leur vigueur, il faut que des lecteurs cultivés, guidés par une connaissance historique aiguë, mènent une archéologie des conditions de lecture et de compréhension des œuvres en leur temps. Ainsi voit-on, contrairement à une idée reçue, que ce n'est pas tant la variabilité des temps - et de la sensibilité des lecteurs - qui impose, cherche et produit une nouvelle «lecture » des œuvres anciennes, c'est intrinsèquement leur inépuisable richesse, dont certains aspects sont, à travers le passage des siècles, plus ou moins saillants. D'autant que «l'anachronisme est une condition herméneutique de toute interprétation » (Normand Doiron, voir bibliographie). La richesse de l'interprétation réside dans la polyphonie du texte, polyphonie d'où provient aussi, paradoxalement, un danger réel de mésintelligence. «Le texte littéraire est par essence voué au malentendu » rappelle Philippe Hamon. Mais qu'à cela ne tienne : l'auteur peut prendre le risque d'être mésinterprété par certains s'il compte sur la subtilité des autres à déchiffrer son ambition cachée.

On peut être tenté de relier l'idée de contrainte avec l'Oulipo et les usages ludiques, de prime abord ostentatoires mais finalement herméneutiques, que ce groupe d'écrivains en a faits. Comme Jean-François Puff l'a précisé, ce serait méconnaître toutefois la distance entre ce que Jacques Rancière appelle le régime représentatif ou poétique et le régime esthétique. Le système poétique de l'époque dite classique a ses spécificités qui justifient l'espace chronologique choisi pour ce recueil d'études. L'analyse récente de Normand Doiron déjà citée a montré le lien entre l'avènement des nouvelles contraintes de l'État monarchique aux XVI ${ }^{\mathrm{e}}$ et $\mathrm{XVII}^{\mathrm{e}}$ siècles et leur traduction en préceptes moraux. Le jeu avec la contrainte littéraire a été, dès le $\mathrm{XVI}^{\mathrm{e}}$ 
siècle, une réponse à la contrainte de la vie de cour. Normand Doiron donne en exemple Le Philosophe de court de Philibert de Vienne (I548), un éloge satirique et qui exprime une attitude morale face à " l'honnête dissimulation » préconisée dans la vie de cour : « la satire, dit-il, est la forme littéraire d'une sagesse de la feinte ».

Un peu comme le fascinant personnage du roman de Siri Hustvedt, Un été sans les hommes, qui au dos de ses sages broderies de nappes de Noël et de coussins décoratifs a cousu des motifs infernaux ou érotiques, nombreux sont les auteurs classiques qui ont joué avec les exigences normées du genre littéraire qu'ils pratiquaient pour avancer, masquées, d'autres intentions, qu'elles soient politiques, religieuses, philosophiques, voire simplement injurieuses : car plus ces intentions sont socialement dangereuses et plus le double jeu a bien sûr besoin de se cacher. Mais cette ambivalence peut aussi être inscrite ouvertement dans les textes sans redouter d'être dénoncée, quand celui qu'elle attaque ou concerne peut faire semblant d'y lire autre chose, de beaucoup plus visible, que ce qui y est subrepticement condamné ou vanté. Ce dernier cas se révèle très lisible dans l'étude où Béatrice Brottier montre la manipulation politicienne inscrite au cœur des éloges funèbres dédiés à Henri IV : la décence interdisant d'interdire des hommages au roi défunt, loisible aux poètes astucieux de subvertir l'hommage attendu en message politique de soutien à la Régente. Cette pratique politico-poétique évoque l'usage que, bien plus tard, les républicains dans la France monarchique du XIX ${ }^{\mathrm{e}}$ siècle, ou les résistants en lutte contre toutes les dictatures du $\mathrm{Xx}^{\mathrm{e}}$ siècle, ont fait des enterrements : ces cortèges difficiles à interdire pour des raisons de décence, là encore, se muent aisément aux yeux des participants en manifestations où ils peuvent se compter et mesurer leurs forces, à la barbe de gouvernements assez entravés à trouver un motif crédible pour interdire ces défilés, face à l'opinion nationale ou internationale. Peut-être d'ailleurs le manque de réaction en cette occurrence est-il une attitude subtile, sachant qu'interdire ces manifestations funèbres serait leur reconnaître une potentielle force de revendication et un contournement réussi de la censure. Cependant le choix laissé au potentiel censeur est, in fine, celui d'avoir l'air ou naïf ou despotique ; la vigueur du piège créé se mesure sur cette seule observation. Toute parée d'une normalité affichée, la parole profonde se libère, dans et à l'abri d'une pratique sociale convenue.

Il est plus aisé, politiquement et même socialement parlant, de regarder comme un jeu sans importance les facéties poétiques que de les censurer. Leur importance peut aussi se loger ailleurs. Sylvain Menant montre que la forme brève de l'épitaphe - socialement très sélective et inscrite dans une longue tradition de sérieux - peut être elle-même l'enjeu de manipulations de la part des auteurs : satirique, philosophique, voire autobiographique, ce tout petit poème qui se doit d'être un éloge du disparu se détourne facile- 
ment en revendication de l'éthos de l'auteur de l'épitaphe, de son droit à se venger ou à dénigrer un adversaire. La forme la plus avancée de ce jeu tient soit au désir de mort qu'elle peut convoyer, à l'égard d'un récipiendaire encore vivant, soit par la forme de pied de nez que constitue structurellement une épitaphe par anticipation quand le poète la compose pour lui-même : comme s'il voulait être certain de se venger outre-tombe ou de confisquer par avance le jugement qui sera porté sur lui post mortem.

Énigmes et épigrammes sont de ces genres qui semblent devoir partager également deux caractères : la brièveté et l'inconséquence. En se penchant sur l'énigme, jeu littéraire traditionnel auquel le journalisme donne, avec un nouveau public, une vitalité qui se maintient tout au long du XvIII ${ }^{\mathrm{e}}$ siècle, Timothée Léchot montre que cette facétie obéit à des règles de convenance et d'honnêteté : les exceptions, et il y en a, qui franchissent ces barrières implicites, sont autant de transgressions à caractère burlesque et provocateur qui attestent que la virtuosité d'une énigme peut contribuer à la faire admettre, malgré un sujet obscène ou ignoble. Il semble, toutefois, que les modes de transgressions ne se superposent pas : Timothée Léchot observe que la forme poétique est d'autant plus conforme que le sujet l'est peu, ou inversement, mais non pas les deux ensemble.

L'art de la polémique implicite se cache aussi là où le lecteur contemporain a du mal à l'imaginer, voire à l'identifier. C'est ce que démontrent, chacun dans leur domaine, Adrien Paschoud et Jean-Luc Chappey.

Adrien Paschoud étudie le discours de réception de Fénelon à l'académie en I693 où, tradition oblige, il fait l'éloge de son prédécesseur, Pellisson : admirer son talent d'écrivain, son style « noble et léger ", révérer au passage Richelieu, fondateur de l'Académie française, sont les thèmes obligés de ce genre du discours de réception. Mais l'élément essentiel de celui-ci, un court paragraphe apparemment stéréotypé en forme d'attestation de catholicité de l'académicien disparu, a une puissante valeur idéologique. L'encomiaste - qui n'avait pas encore rédigé les grandes œuvres qui font aujourd'hui sa réputation, ni encouru la disgrâce du roi pour son présumé quiétisme qui lui valut l'archevêché de Cambrai, forme élégante d'exil - était alors connu pour ses talents de pédagogue, notamment à l'égard des convertis. Il bat en brèche, dans ce bref passage, les rumeurs qui voudraient que la mort sans sacrements de Pellisson signifiât une adhésion intime au protestantisme, malgré sa conversion au catholicisme qui remonte à I670. Fénelon affirme - brièvement mais éloquemment, avec une hypotypose où le vieil académicien se traîne " encore au pied des autels jusqu'à la veille de sa mort » - qu'il se préparait au-delà de ses forces humaines à la fois à son ouvrage sur l'Eucharistie et à " recevoir les sacrements avec ferveur ", pluriel poétique si l'on veut, ou pluriel ambigu qui peut englober parmi d'autres celui de l'extrême-onction, alors étonnamment relativisé. L'inachèvement de son 
œuvre de théologien rejoignant sa mort sans sacrements, l'éloge littéraire et l'éloge moral se conjuguent au service de la mémoire de Pellisson, dans une campagne d'opinion en faveur de son orthodoxie.

Jean-Luc Chappey, en détaillant sur plus d'un demi-siècle l'évolution des usages d'édition dans les dictionnaires biographiques, montre combien ces textes apparemment factuels, documentaires et informatifs sont travaillés par une intense volonté polémique. L'admission ou le rejet des auteurs dans ce panthéon avant la lettre délimite en effet les normes élaborées par l'auteur de chaque dictionnaire, y compris ses choix théologiques. D'où l'exigence qui en découle, pour l'historien de la littérature, de repérer les stratégies de promotion ou de dénigrement qui font accepter ou non tel ou tel auteur dans un ouvrage de ce genre. La multiplicité de ces types de dictionnaires et la fréquence de leurs rééditions expriment sans doute - autant que leur indéniable succès commercial - non seulement la vigueur de la lutte d'influence qu'elles traduisent et relancent à chaque nouveau tirage, mais encore leur désir de modeler, de la République des lettres, l’image qui convient au compilateur. Malgré son apparence anodine pour le profane, la forme de dictionnaire est propice à toutes les audaces : Bayle et l'Encyclopédie en sont des exemples criants et bien connus, Jean-Luc Chappey rappelle que cela concerne toutes les formes de dictionnaires, notamment biographiques.

En s'intéressant à deux discours de réception de Lezay-Marnésia à l'académie de Besançon, Julie Lablanche prouve que ce genre apparemment très conformiste et codé est susceptible de laisser beaucoup d'audace, du moins à une plume alerte. Reçu comme associé en I777 puis comme titulaire en $\mathrm{I} 778$, le marquis a deux fois l'occasion de se singulariser. Une première fois, il sait infléchir les thèmes (convenus) d'un discours de réception en saluant l'assistance, militaire par exception, pour rendre hommage à sa formation non-académique ; or la force des attentes est telle, dans le domaine académique, que ce qui manque à un discours de réception (ici l'éloge de la vie académique et celui du fondateur de l'académie de Besançon, le duc de Tallard) parle plus haut que ce qui s'y trouve. Pour sa deuxième réception, il fait l'éloge de D'Alembert, de l'Académie française, et critique tout aussi ouvertement le manque d'originalité des académiciens comtois. Par manque de compte rendu dans les registres, on ignore comment ce discours fut reçu. Du moins constate-t-on que Marnésia osa le prononcer et qu'il figure dans les archives de l'académie de Besançon : c'est l'effet de série et la distance entre ce discours et celui que ses confrères étaient en droit d'attendre qui permet d'en mesurer l'originalité et qui en fait pour nous tout le sel.

C'est naturellement l'existence de la censure qui est la plus grande pourvoyeuse de ces jeux, quand ils sont littéraires. Morales, sociales, religieuses, politiques, les sources et causes de censure ou d'auto-censure sont variées, mais il est indéniable qu'on leur doit un renchérissement de subtilité. En 
portant une attention précise au corpus des prospectus d'édition réuni par Adrien Beuchot, éditeur acharné des œuvres de Voltaire sous la Restauration, Nicolas Morel montre que la forme courte de la citation peut devenir profondément emblématique : peut-on censurer quelques lignes de Voltaire données en exemple typographique de la qualité d'une édition proposée à la souscription? En théorie oui, selon les lois en vigueur sous la Restauration. Mais le choix judicieux de ces quelques lignes à visée publicitaire permet, d'un air faussement innocent, de lancer un message potentiellement vigoureux sur le ton qu'aura l'édition proposée, message adressé même aux lecteurs qui ne souscriraient pas : soit une vraie campagne d'opinion.

Pour les écrivains, la contrainte n'est ni une servitude ni la route étroite qui mène au conformisme. Elle peut en avoir l'air, aux yeux des lecteurs trop littéralistes par exemple de Boileau, qui envisagent ses préceptes comme des interdits castrateurs; mais son propre talent de satiriste montre que Boileau lui-même sait ruser avec les règles, et pas seulement dans le domaine de la satire. Sa persistante célébrité émane assurément de son usage scolaire, et cet usage même dérive de la limpidité de ses vers. Mais plutôt qu'un poème fondateur ou initiateur, son Art poétique (I674) est un résumé ou un aboutissement d'un siècle de poétique, il n'est que de se rappeler que La Poétique de Scaliger, qui, malgré sa rédaction en latin, fonde la critique notamment dramatique en français, date de I56I et mérite bien plutôt cette place de pionnier souvent attribuée à Boileau. Même si elle sert en premier lieu à censurer tout ce qui n'appartient pas au « bon goût » (social ou littéraire) et beaucoup, on le sait, a été rejeté hors de son domaine au XVII ${ }^{\mathrm{e}}$ siècle, la contrainte est paradoxalement aussi un ressort de la créativité. Michel Jeanneret a montré la valeur théologique - critique il va sans dire - de certains poèmes ouvertement obscènes attribués à Malherbe ; inversement, si l'on peut dire, Antony McKenna a montré quelle lecture théologique on pouvait faire des pièces de Molière, où la critique des médecins sert de drapé et de paravent à une critique de la rigueur janséniste. Il ne s'agit pas tant de stéganographie que de prudence. En effet à cause de sa nature foncièrement libertaire, le jeu avec les traditions et les codes reste un jeu dangereux. Par le masque ou le détour, l'écrivain affirme, au cœur même de pratiques littéraires conformes en apparence, sa liberté, dont l'efficace est certes suspendue à la subtilité du lecteur mais dont le plaisir est déjà intimement présent - on n'en peut douter - au stade de la création textuelle.

Il est dans la nature de la littérature d'être libre. Même apparemment asservie soit aux usages - qu'ils soient sociaux ou génériques - soit aux puissances, elle a la subtilité de suggérer en filigrane, par antiphrase, par allusion, tout autre chose que ce qu'elle énonce. Car la littérature use à son profit de toutes les possibilités de distorsion de sens que lui offre la langue. Il y a matière à méditation sur la distance entre langue naturelle et codes : la sub- 
tilité que son usage artistique et cultivé peut donner à la langue naturelle ne permet pas de l'encager dans un code. La littérature, lue par un esprit vivant, offre un sens toujours à réinventer, ouvrant sur des interprétations chaque fois réactualisées. D'aucuns peuvent, par aveuglement, regretter l'imperfection de la langue naturelle qu'il faudrait plutôt appeler son inachèvement, sa mutabilité, sa vie en un mot. L'histoire montre que la subtilité de la littérature, se faufilant dans tous les interstices, se contorsionnant dans toutes les postures, faisant grimacer tous les masques lui a permis, très souvent, de résister aux censures, de les contourner, de les transgresser. Nous avons besoin de pouvoir compter sur cette force vitale.

\section{Bibliographie}

Bénichou, Paul, « Réflexions sur la critique littéraire », Variétés critiques, Paris, Corti, I996, pp. 27I-292.

Bonnier, Xavier, "Un mort qui toujours vif sera” : Marot et l'étrange épitaphe de Pierre de Villiers ", Réforme, Humanisme, Renaissance, 84, 20I7, pp. 7-24.

Bourahla, Aziza, "Par un secret subtil noblement inventé" : Babel ou la Tour renversée dans le Microcosme de Scève ", Réforme, Humanisme, Renaissance, 74, 20I2, pp. 4I-59.

Bowen, Barbara, «Agrippa's De vanitate : Polemic or Paradox ? ", Bibliothèque d'Humanisme et Renaissance, 34, I972, pp. 249-256.

Cavaillé, Jean-Pierre, « Ruser sans mentir : de la casuistique aux sciences sociales. Le recours à l'équivocité, entre efficacité pragmatique et souci éthique ", Les Raisons de la ruse. Une perspective anthropologique et psychanalytique, Paris, La Découverte, « Recherches/MAUSS », 2004, pp. 93-II6.

Cesbron, Georges (éd.), Du Bellay. Actes du colloque International d'Angers, Angers, PU Angers, 1990.

Chaigne, Dominique, "S'éloigne[r] du sens littéral, pour s'approcher de mes intentions" ou l'art de l'oblique dans Les Femmes illustres ou Les Harangues héroïques de Georges et Madeleine de Scudéry ", éd. Caroline Jacot-Grapa et Guyonne Leduc, Dire sans dire : stratégies obliques. Actes du colloque de Lille, 20II, http://I7I8.fr/dire-sans-dire-strategis-obliques/ [consulté le 9 avril 20I8].

Chartier, Roger, Le Jeu de la règle. Lectures, Bordeaux, PU de Bordeaux, 2000. Compagnon, Antoine, La Seconde Main, ou le travail de la citation, Paris, Seuil, I979.

Degen, Guy, «Autour d'un lit de cendres : l'image de Louis IX et de sa seconde croisade dans les panégyriques de saint Louis aux XVII ${ }^{\mathrm{e}}$ et XVIII ${ }^{\mathrm{e}}$ siècles ", La Littérature et ses avatars. Discrédits, déformations et réhabilita- 
tions dans l'histoire de la littérature, dir. Yvonne Bellenger, Paris, Klincksieck, I99I, pp. I26-I49.

Desan, Philippe, « De la poésie de circonstance à la satire : Du Bellay et l'engagement poétique », Du Bellay. Actes du colloque International d'Angers, Angers, PU Angers, I990, pp. 42I-438.

Doiron, Normand, «La Contrainte de la cour », Poétique, I78/2, 2015, pp. I63I82.

Domenech, Jacques (dir.), Censure, autocensure et art d'écrire de l'Antiquité à nos jours, Paris, Éditions Complexes, 2005.

Fontaine, Marie-Madeleine, "Des mots à la rime et de leur raison ", Du Bellay. Actes du colloque International d'Angers, Angers, PU Angers, I990, pp. 26I-284.

Gavarini, Fausta, « Montanus pro domo sua (à propos de Essais II, I : De I'inconstance de nos actions) ", L'Esprit et la lettre. Mélanges Jules Brody, éd. Louis van Delft, Tübingen, Gunter Narr Verlag, I99I, pp. 2I-35.

Gendre, André, «Entre poésie allégorique, éloge et pamphlet : Anne de Marquets et le colloque de Poissy ", Devis d'amitié. Mélanges Nicole Cazauran, éd. Jean Lecointe, Catherine Magnien, Isabelle Pantin, Marie-Claire Thomine, Paris, Champion, 2002, pp. 579-595.

Hallyn, Fernand, « Le Songe de Du Bellay : de l'onirique à l'ironique ", Du Bellay. Actes du colloque International d'Angers, Angers, PU Angers, I990, pp. 3OI-3I2.

-. «La devise anagrammatique, culte et haine du pouvoir », dir. Luisa Rotondi Secchi Tarugi, Cultura e potere nel Rinascimeno. Atti del Convegno Chianciano-Pienza I997, Firenze, Cesati, 20I4, pp. 575-588.

Hamon, Philippe, L'Ironie littéraire : essai sur les formes de l'écriture oblique, Paris, Hachette, I996.

Hellegouarc'h, Jacqueline, "Quelques mots clins d'œil chez Voltaire », Le Siècle de Voltaire, hommage à René Pomeau, éd. Ch. Mervaud et S. Menant, Oxford, Voltaire Foundation, 1987, t. II, pp. 537-544.

Hummel, Pascale (dir.), Paralangues : études sur la parole oblique, Paris, Philologicum, 2 oro.

Jacot-Grapa, Caroline et Leduc, Guyonne, Dire sans dire : stratégies obliques. Actes du colloque de Lille, 20II, http://I7I8.fr/dire-sans-dire-strategisobliques/ [consulté le 9 avril 20I8].

Jeanneret, Michel, «Sonnets luxurieux : de l'érotique à l'obscène ", Les Fruits de la Saison, Mélanges André Gendre, éd. Marie-Jeanne Liengme Bessire, Loris Petris, Philippe Terrier, Genève, Droz, 200o, pp. 385-400.

-. Éros rebelle. Littérature et dissidence à l'âge classique, Paris, Seuil, 2003.

Laurens, Pierre, L'Abeille dans l'ambre. Célébration de l'épigramme de l'époque alexandrine à la fin de la Renaissance, Paris, Les Belles-Lettres, 1989, rééd. 2012. 
McKenna, Antony, Molière dramaturge libertin, Paris, Champion, 2005.

Mervaud, Christiane, "Variations sur le premier homme : une série d'articles "Adam" dans quelques dictionnaires du XVIII" siècle ", Séries et variations. Études littéraires offertes à Sylvain Menant, dir. Luc Fraisse, Paris, PUPS, 2006, pp. 505-5I4.

Miernowski, Jan, Signes dissimilaires. La quête des noms divins dans la poésie française de la Renaissance, Genève, Droz, 1997.

Pot, Olivier, « Osons être des littéraires », Versants, 63/I, 20I6, pp. 99-II2.

Puff, Jean-François, « La contrainte et la règle », Poétique, 2004/4, pp. 455-465. Rancière, Jacques, Le Partage du sensible, Paris, La Fabrique Éditions, 2000.

Rousset, Jean, La Littérature de l'âge baroque. Circé et le paon, Paris, Corti, 1953. Sangsue, Daniel, La Relation parodique, Paris, Corti, 2007.

Schoentjes, Pierre, Recherche sur lironie et ironie de la recherche, Gent, Rijksuniversiteit Gent, 1993.

Starobinski, Jean, Les Mots sous les mots, Paris, Gallimard, I97I.

Staub, Hans, Le Curieux Désir : Scève et Peletier du Mans, poètes de la connaissance, Genève, Droz, 1967.

Thomine, Marie-Claire, "Les Jeux sur le poncif dans La Nouvelle Fabrique des excellents traicts de vérité de Philippe d'Alcripe », Devis d'amitié. Mélanges Nicole Cazauran, éd. Jean Lecointe, Catherine Magnien, Isabelle Pantin, Marie-Claire Thomine, Paris, Champion, 2002, pp. 239-263.

Weber, Henri, « Poésie polémique et satirique de la Réforme sous les règnes de Henri II, François II et Charles IX », Cahiers de l'Association internationale des études francaises, I0, I958, pp. 89-II8.

Weerdt-Pilorge, Marie-Paule de, « Une grande entreprise éditoriale de la Restauration : la collection des Mémoires relatifs à la Révolution française de Berville et Barrière ", Cahiers de l'Association internationale des études françaises, 67, 20I5, pp. 311-323.

Wild, Francine (dir.), Le Sens caché. Usages de l'allégorie du Moyen Âge au XVII siècle, Arras, Artois Presses Université, 2013. 
